\title{
EXPRESSIBILITY OF PROPERTIES OF RELATIONS
}

\author{
HAJNAL ANDRÉKA, IVO DÜNTSCH, AND ISTVÁN NÉMETI
}

\begin{abstract}
We investigate in an algebraic setting the question in which logical languages the properties integral, permutational, and rigid of algebras of relations can be expressed.
\end{abstract}

\section{INTRODUCTION}

The question, which properties of relations can be expressed in which logical languages is of considerable interest in the fields of (finite) model theory and descriptive complexity, and the reader is invited to consult [Fag87], [Imm82] for more detailed information. Among the problems in this area are those which are concerned with the automorphisms of a given binary relation; typical questions include

(1) Does $R$ have a non trivial automorphism?

(2) Is the group of automorphisms of $R$ transitive?

We shall investigate these and other problems from an algebraic (and sometimes more abstract) point of view using Tarski's relation algebras and cylindric algebras. Roughly speaking, a relation algebra is a description of how various relations must interact among each other. More concretely, given a set $\bar{R}=\left\{R_{i}: i<n\right\}$ of binary relations on a set $U$, we can form the closure of this set under the Boolean operations, composition of relations, and converse, and add the identity as an extra constant; the result will be an algebra $\mathfrak{A}$ of binary relations (BRA). Since the operations used are first order definable, any automorphism of the first order structure $\langle U, \bar{R}\rangle$ will preserve all the relations in A as well. Such an automorphism will be called a base automorphism of the algebra $\mathfrak{A}$ (We use the qualified term to distinguish them from the automorphisms of the algebra). A fundamental result by Tarski states that $\mathfrak{A}$ contains exactly those binary relations on $U$ which are definable in $\langle U, \bar{R}\rangle$ by first order formulas having at most three variables. The algebras corresponding to $n$ variables (and $n$-ary relations) are the cylindric algebras of dimension $n$. Thus, the equational logic of relation algebras (cylindric algebras of dimension $n$ ) corresponds roughly to the three $(n)$ variable fragment of full first order logic. Logic with restricted resources has been considered among others in [Imm82], [IK89].

Conversely, if we are given an abstract relation algebra $\mathfrak{B}$ (to be defined below), and a BRA $\mathfrak{A}$ on a set $U$ such that $\mathfrak{A}$ is isomorphic to $\mathfrak{B}$ (also called a representation of $\mathfrak{B}$ ), then the algebraic structure of $\mathfrak{B}$ (or, equivalently, of $\mathfrak{A}$ ) prescribes how the concrete relations in $\mathfrak{A}$ must interact with respect to the Boolean operations, composition, and converse. Thus, instead of looking at the properties of a given set $\bar{R}$ of concrete relations, we can look at the isomorphism type of the relation algebra $\mathfrak{A}$ which is generated by $\bar{R}$, and ask whether we can say something about

Date: J Symb Logic 60 (1995), 970-991.

1991 Mathematics Subject Classification. 03G15. 
the algebraic structure of $\mathfrak{A}$ which will imply an answer for our question regarding $\bar{R}$. For example, we show below that the property that a relation algebra $\mathfrak{B}$ has a a representation with a transitive group of base automorphisms is a general first order property by giving a set of axioms which describe this property. From the form of these axioms it follows that in the concrete case, a first order structure $\langle U, \bar{R}\rangle$ of binary relations has a transitive group of automorphisms if and only if no proper nonempty subset is definable in the model, and, furthermore, that this property is not expressible in $L_{\omega_{1} \omega}^{\omega}$, the finite variable fragment of $L_{\omega_{1} \omega}$. We also generalise most of the results to $n$-ary relations.

Rigidity, i.e. the property of a structure $\langle U, \bar{R}\rangle$ of having no nontrivial automorphisms, is diametrically opposed to having a transitive group of automorphisms: While in the latter case no proper nonempty subset is definable in the model, in a finite rigid structure every subset is definable. We shall exhibit for every $n<\omega$ a rigid symmetric binary relation $R$ on a finite set $U$ with the property that no proper nonempty subset of $U$ is definable in the model $\langle U, R\rangle$ by a first order formula with at most $n$ variables. The construction will be a concrete example for the fact that rigidity is not expressible in $L_{\omega_{1} \omega}^{\omega}$. We also show by a concrete example that non - rigidity is not expressible in the fragment of second order logic where the first order part is in the Bernays - Schönfinkel prefix class, and, with the help of a result by $\mathrm{N}$. Immerman, that rigidity cannot be expressed in monadic second order logic, even if a built in linear order is allowed. It is also proved that for any rigid relation $R$ on a set $U$ having no more than seven elements, every subset of $U$ is definable in the model $\langle U, R\rangle$ by a formula using no more than three variables.

\section{DEFINITIONS AND NOTATION}

If $U$ is a set, $|U|$ denotes the cardinality of $U$. We identify $\{0, \ldots, n-1\}$ with the ordinal $n$, and sometimes emphasise this by writing $\mathbf{n}$. If no confusion is likely to arise, algebras are referred to by their respective carrier set. For an ordinal $\alpha,{ }^{\alpha} U$ denotes the set of all $\alpha$-termed sequences of elements of $U$.

2.1. Logics. We assume that the reader is familiar with the basic facts of first or second order logic. Our first order (FO) languages consist of predicate symbols, logical connectives $\wedge, \neg$, the existential quantifier $\exists$ and equality, and we use the usual abbreviations. When considering relational structures $\langle U, R\rangle$ as first order models, we tacitly assume that an appropriate first order language $L$ is given. For notational reasons, we shall sometimes identify predicate symbols with the predicates which interpret them, when no confusion is likely to arise. If $\varphi(x)$ is a formula with the free variable $x$, and $\langle U, R\rangle$ is a model of the language $L$, then the truth set of $\varphi(x)$ in the model $\langle U, R\rangle$ is the set

$$
\operatorname{def} \varphi=\{a \in U:\langle U, R\rangle \models \varphi(x / a) .
$$

If $M \subseteq U$ and $M=\operatorname{def} \varphi$ for some $\varphi$, then we say that $M$ is definable in the model $\langle U, R\rangle$. Similarly, we extend this definition to languages with more than one predicate symbol and formulas with more than one free variable. The symbols $\bigvee$ and $\bigwedge$ will be used for infinite disjunction, resp. conjunction, of formulas.

If $L$ is a first order language, $L_{n}$ denotes its $n$-variable fragment; in other words, $L_{n}$ has the same logical and predicate symbols as $L$, but only the variables $v_{0}, \ldots, v_{n}$. 
$L_{\omega_{1} \omega}$ is the extension of first order logic which allows infinite disjunctions and conjunctions, but only finite quantifier depth. For $1 \leq k \leq \omega$ let $L_{\omega_{1} \omega}^{k}$ be the fragment of $L_{\omega_{1} \omega}$ which consists of all formulas with at most $k$ distinct variables, and set

$$
L_{\omega_{1} \omega}^{\omega} \stackrel{\text { def }}{=} \bigcup\left\{L_{\omega_{1} \omega}^{k}: 1 \leq k<\omega\right\} .
$$

This is also called the finite variable fragment of $L_{\omega_{1} \omega}$.

Second order logic (SO) is the enhancement of FO by allowing quantification over predicates. We shall be considering various fragments of SO:

The fragment of SO where quantification is allowed over unary predicates only is called monadic second order logic (MSO).

$\Sigma_{1}^{1}$ formulas have the form

$$
\left(\exists P_{0}\right)\left(\exists P_{1}\right) \ldots\left(\exists P_{n}\right) \psi,
$$

where $P_{0}, \ldots, P_{n}$ are predicate variables, and $\psi$ is a first order formula in the language enhanced by $P_{0}, \ldots, P_{n}$. Similarily, $\Pi_{1}^{1}$ formulas have the form

$$
\left(\forall P_{0}\right)\left(\forall P_{1}\right) \ldots\left(\forall P_{n}\right) \psi \text {. }
$$

A $\Sigma_{1}^{1} \Pi_{2}^{0}$ formula is a $\Sigma_{1}^{1}$ formula whose first order part is equivalent to a prenex $(\forall \bar{x})(\exists \bar{y})$ formula.

$L^{<}$is the language $L$ enhanced by a binary predicate $<$ which on every structure with base set $U=\{0, \ldots, n\}$ is to be interpreted as the natural order on $U$.

2.2. Groups. The symmetric group on $U$ is denoted by $\operatorname{Sym}(U)$. If $G$ is a subgroup of $\operatorname{Sym}(U)$ - a fact which we write as $G \leq \operatorname{Sym}(U)$ - , and if $M \subseteq U$, we denote by $M^{G}$ the set $\{\varphi(x): x \in M, \varphi \in G\}$; for $\varphi \in \operatorname{Sym}(U), M^{\varphi}$ is the set $\{\varphi(x): x \in M\}$. A fixed block of $G$ is a nonempty subset $M$ of $U$ such that $M=M^{G}$; a minimal fixed block is called an orbit of $G$. If $G$ has only one orbit, it is called transitive.

$G$ is called semiregular if the identity is the only element of $G$ that fixes a point, or, equivalently, if for all $\varphi, \psi \in G$, the fact that $\varphi(x)=\psi(x)$ for some $x \in U$ implies that $\varphi=\psi$. If $G$ is semiregular and transitive, then it is called regular.

2.3. Relation algebras. A relation algebra ${ }^{1}(\mathrm{RA})$

$$
\left\langle A,+, \cdot,-, 0,1, \circ,^{-1}, 1^{\prime}\right\rangle
$$

is a structure of type $\langle 2,2,1,0,0,2,1,0\rangle$ which satisfies

(1) $\langle A,+, \cdot,-, 0,1\rangle$ is a Boolean algebra.

(2) $\left\langle A, \circ,{ }^{-1}, 1^{\prime}\right\rangle$ is an involuted monoid.

(3) For all $a, b, c \in A$ the following conditions are equivalent:

$$
(a \circ b) \cdot c=0,\left(a^{-1} \circ c\right) \cdot b=0,\left(c \circ b^{-1}\right) \cdot a=0 .
$$

The full algebra of binary relations

$$
\operatorname{Rer}(U) \stackrel{\text { def }}{=}\left\langle\operatorname{Rel}(U), \cup, \cap,-, \emptyset,{ }^{2} U, \circ,{ }^{-1}, 1^{\prime}\right\rangle
$$

is a relation algebra, where $\operatorname{Rel}(U)$ is the set of all binary relations on $U, \cap, \cup,-$ are the usual set theoretic operations, and $\emptyset,{ }^{2} U$ are, respectively, the empty and the universal relation, $\circ$ is relational composition, ${ }^{-1}$ the relational inverse (i.e.

\footnotetext{
${ }^{1}$ The interested reader is invited to consult [TG87] for the background of relation algebras.
} 
$\left.P^{-1}=\{\langle x, y\rangle:\langle y, x\rangle \in P\}\right)$, and $1^{\prime}$ is the identity relation on $U$. We shall usually use $P, Q, R, \ldots$ to denote binary relations on $U$. A subset $A$ of $\operatorname{Rel}(U)$ which is closed under the distinguished operations of $\mathfrak{R e l}(U)$ and contains the distinguished constants is called an algebra of binary relations (BRA) on $U$, usually denoted by $\mathfrak{A}$. It is a subalgebra of $\mathfrak{R e l}(U)$, a fact which we denote by $\mathfrak{A} \leq \mathfrak{R e l}(U)$. If $\mathfrak{A} \leq \mathfrak{R e l}(U)$, then $A t(\mathfrak{A})$ denotes the set of atoms of (the Boolean part of) $\mathfrak{A}$. A complete and atomic $\mathfrak{A}$ is completely determined by the relation composition table of $A t(\mathfrak{A})$. When writing such a table, we usually omit column and row $1^{\prime}$, if $1^{\prime}$ is an atom of $\mathfrak{A}$. If $R_{0}, \ldots, R_{k} \in \operatorname{Rel}(U)$, we denote the BRA generated by $R_{0}, \ldots, R_{k}$ by $\left\langle R_{0}, \ldots, R_{k}\right\rangle$.

A relation algebra $\mathfrak{A}$ is called representable if it is isomorphic to a subalgebra of a product of full algebras of binary relations.

The following fundamental result is due to A. Tarski [TG87]:

Proposition 2.3.1. If $R_{0}, \ldots, R_{k} \in \operatorname{Rel}(U)$, then $\left\langle R_{0}, \ldots, R_{k}\right\rangle$ is the set of all binary relations on $U$ which are definable in the relational structure $\left\langle U, R_{0}, \ldots, R_{k}\right\rangle$ by first order formulas using at most 3 variables.

For $P, Q \in A \leq \mathfrak{R e l}(U)$ and $x, y, z \in U$ we usually write $x P y$ if $\langle x, y\rangle \in P$, and $x P y Q z$ means $x P y$ and $y Q z$. We also set

$$
\begin{aligned}
\operatorname{dom}(P) & =\{x \in U: \text { There is some } y \in U \text { such that } x P y\} \\
\operatorname{ran}(P) & =\{x \in U: \text { There is some } y \in U \text { such that } y P x\} \\
\operatorname{dom}_{P}(x) & =\{y \in U: y P x\} \\
\operatorname{ran}_{P}(x) & =\{y \in U: x P y\} .
\end{aligned}
$$

The set $\operatorname{ran}_{P}(x)$ is also called a row of $P$. If $\left|\operatorname{ran}_{P}(y)\right|=\left|\operatorname{ran}_{P}(x)\right|$ for all $x, y \in \operatorname{dom}(P)$, then $P$ is called regular.

If $\mathfrak{A}$ is any RA, then $x \in A$ is called a functional element if $x^{-1} \circ x \leq 1^{\prime} . \mathfrak{A}$ is called integral if, for all $x, y \in A, x \circ y=0$ implies $x=0$ or $y=0$. A well known characterization of integral RAs [JT52] is given by

Lemma 2.3.2. Let $\mathfrak{A}$ be a relation algebra. Then, the following statements are equivalent:

(1) $\mathfrak{A}$ is integral.

(2) $1^{\prime}$ is an atom of $\mathfrak{A}$.

(3) $x \circ 1=1$ for any non zero $x \in A$.

(4) Every functional non zero $x \in A$ is an atom of $\mathfrak{A}$.

(5) If $\mathfrak{A}$ is a BRA on a finite set $U$, then $\operatorname{dom}(R)=\operatorname{ran}(R)=U$ for some atom $R$ of $\mathfrak{A}$.

If $\mathfrak{A} \leq \mathfrak{R e l}(U)$ and $E \in A$ is an equivalence relation, then $B \stackrel{\text { def }}{=}\{R \in A$ : $R \subseteq E\}$ becomes a BRA on $M \stackrel{\text { def }}{=} \operatorname{dom}(E)$ under the operations $\cup, \cap, \emptyset, \circ,-1$ inherited from $\mathfrak{A}$, greatest element $E$, identity element $\{\langle x, x\rangle: x \in M\}$, and complementation being relative to $E$. This algebra is called the relative algebra of $\mathfrak{A}$ with respect to $E$. Unless $E={ }^{2} U$, it is not a subalgebra of $\mathfrak{A}$.

Suppose that $\mathfrak{A}$ is not integral; then, there are atoms $E_{i}, i<k$, of $\mathfrak{A}$ such that $E_{i} \subseteq 1^{\prime}$ for $i<k$. For $i, j<k$, set $U_{i}=\operatorname{dom}\left(E_{i}\right)$ and $U_{i j}=U_{i} \times U_{j}$. We observe that $U_{i j}=E_{i} \circ{ }^{2} U \circ E_{j}$, so that $U_{i j} \in A$. Since each $U_{i i}$ is an equivalence 
relation on $\operatorname{dom}\left(E_{i}\right)$ contained in $\mathfrak{A}$, we can consider the relative algebra of $\mathfrak{A}$ with respect to $U_{i i}$, which we denote by $A_{i}$. If $R \in A_{i}$, then $R$ is an atom of $A_{i}$ if and only if $R$ is an atom of $\mathfrak{A}$. Consequently, each $A_{i}$ is an integral relation algebra, and, in loose analogy to permutation groups, we call the algebra $A_{i}$ an integral constituent of $\mathfrak{A}$ and $U_{i}$ its constituent set.

If $\varphi \in \operatorname{Sym}(U)$ and $x, y \in U$, then we set $\varphi\langle x, y\rangle=\langle\varphi(x), \varphi(y)\rangle$, and $R^{\varphi}=$ $\{\varphi\langle x, y\rangle:\langle x, y\rangle \in R\}$; note that $R^{\varphi}=\varphi^{-1} \circ R \circ \varphi$. If $R^{\varphi}=R$, then $\varphi$ is called a base automorphism of $R$. For $\mathfrak{A} \leq \mathfrak{R e l}(U)$ we set

$$
A^{\rho}=\left\{\varphi \in \operatorname{Sym}(U): R^{\varphi}=R \text { for all } R \in A\right\} .
$$

It is not hard to see that $A^{\rho}$ is a subgroup of $\operatorname{Sym}(U)$, called the group of base automorphisms of $\mathfrak{A}$, and that $\varphi \in A^{\rho}$ if and only if $\varphi$ commutes with every atom of $\mathfrak{A}$. If $A=\langle R\rangle$, we also write $R^{\rho}$ isnstead of $\langle R\rangle^{\rho}$.

An integral $\mathfrak{A} \leq \mathfrak{R e l}(U)$ is called c-permutational, if $A^{\rho}$ is transitive. A relation algebra is permutational, if it is isomorphic to a c-permutational one.

Conversely, if $G$ is a subgroup of $\operatorname{Sym}(U)$ and $x, y \in U$, we set

$$
G(x, y)=\{\varphi\langle x, y\rangle: \varphi \in G\},
$$

and let $G^{\sigma}$ be the BRA on $U$ generated by $\{G(x, y): x, y \in U\}$. Observe that the sets $G(x, y)$ are just the orbits of the action of $G$ on ${ }^{2} U$, and hence a partition of ${ }^{2} U$. Indeed, each $G(x, y)$ is an atom of $G^{\sigma}$, and every atom of $G^{\sigma}$ has this form. The assignments $\rho$ and $\sigma$ form a Galois connection, and $\mathfrak{A} \leq \mathfrak{R e l}(U)$ is called Galois closed if $\mathfrak{A}^{\rho \sigma}=\mathfrak{A}$; similarly, $H \leq \operatorname{Sym}(U)$ is Galois closed if $H^{\sigma \rho}=H$ (see [Jón84])

Lemma 2.3.3. Let $\mathfrak{A}$ have the integral constituents $A_{i}, i<k$.

(1) If $P \in A t(\mathfrak{A})$ and $\left|\operatorname{ran}_{P}(x)\right|=1$ for some $x \in U$, then $\left|\operatorname{ran}_{P}(x)\right|=1$ for all $x \in \operatorname{dom}(P)$.

(2) Each $U_{i}$ is a fixed block of $A^{\rho}$. If $M$ is a union of constituent sets, $B$ is the relative subalgebra of $\mathfrak{A}$ with respect to ${ }^{2} M$, and $\varphi \in A^{\rho}$, then the restriction $\psi$ of $\phi$ to $M$ is a base automorphism of $B$.

(3) Suppose that $i, j<k,\left|U_{i}\right| \leq 3,\left|U_{i}\right|<\left|U_{j}\right|,\left|U_{j}\right|$ is not a multiple of $\left|U_{i}\right|$, and that every $R \in A t\left(\mathfrak{A}_{j}\right)$ is regular. Then, $U_{i j} \in A t(\mathfrak{A})$.

(4) Suppose that $k>1, M=U \backslash U_{i}$ for some $i<k$, and let $B$ be the relative subalgebra of $\mathfrak{A}$ with respect to ${ }^{2} M$. If $\varphi \in A_{i}^{\rho}$ and $\psi \in B^{\rho}$, then $\varphi \cup \psi \in A^{\rho}$, if $U_{i j} \in A t(\mathfrak{A})$ for all $j \neq i$.

Proof. 1. Suppose that $\operatorname{ran}_{P}(x)=\{a\}$, and assume that $\left|\operatorname{ran}_{P}(y)\right| \geq 2$ for some $y \in U$. Let $\varphi(x)$ be the formula

$$
(\exists y)(\forall z)[x P y \wedge(x P z \rightarrow y=z)] .
$$

The truth set $M$ of $\varphi$ in the model $\langle U, P\rangle$ is the set of all those elements of $\operatorname{dom}(P)$ whose $P$ - range consists of exactly one element. By our assumption we have $\emptyset \subsetneq M \subsetneq \operatorname{dom}(P)$, and by 2.3.1 the relation $1^{\prime} \cap{ }^{2} M$ is an element of $\langle P\rangle \subseteq A$, since $\varphi$ contains only three variables. This contradicts the fact that $P$ is an atom of $\mathfrak{A}$.

2. If $k=1$, then $\mathfrak{A}$ is integral and $U_{0}=U$ which is clearly a fixed block of $A^{\rho}$. Otherwise, let $k>1$ and assume there are $x \in U_{i}, y \in U_{j}, i \neq j$, and some 
$\varphi \in A^{\rho}$ such that $\varphi(x)=y$. Then, $\langle x, x\rangle \in E_{i}$, but $\langle x, x\rangle \notin \varphi \circ E_{i} \circ \varphi^{-1}$, contradicting that $\varphi$ is a base automorphism of $\mathfrak{A}$.

For the second part, first note that $\psi \in \operatorname{Sym}(M)$, since $M$ is a fixed block of $A^{\rho}$. If $R \in B$, then also $R \in A$ and $R^{\psi}=R^{\varphi}=R$.

3. Assume that for some $i \neq j$ there is some $P \in A t(\mathfrak{A})$ such that $P \subsetneq U_{i j}$, and set $Q=U_{i j} \backslash P$. Let $y \in U_{j}$; if there is exactly one $x \in U_{i}$ with $x P y$, then $P^{-1}$ is a function by the fact that it is an atom and 1 . above. Thus, the $P$-ranges of elements of $U_{i}$ partition $U_{j}$, and from the condition on $A_{j}$ and 2.3.2 it follows that the classes of this partition have the same number of elements. This, however, contradicts that $\left|U_{j}\right|$ is not a multiple of $\left|U_{i}\right|$. Therefore, each $y \in U_{j}$ appears in at least two rows of $P$, and the same arguments apply to $Q$. Hence, for each $y \in U_{j}$ we have $2 \leq\left|\operatorname{dom}_{P}(y)\right| \leq\left|U_{i}\right|-2$, which contradicts $\left|U_{i}\right| \leq 3$.

4. Suppose that $P \in A t(\mathfrak{A})$, and set $\xi=\varphi \cup \psi$. We need to show that $\xi \circ P=P \circ \xi$. If $P \in B$, then

$$
\xi \circ P=\psi \circ P=P \circ \psi=P \circ \xi .
$$

Similarily, if $P \in A_{i}$, then

$$
\xi \circ P=\varphi \circ P=P \circ \varphi=P \circ \xi .
$$

Otherwise, let $P=U_{i j}$ for some $j \neq i$. Then,

$$
\xi \circ P=\varphi \circ P=P \circ \psi=P \circ \xi:
$$

Just observe that $\operatorname{ran}_{p}(x)=U_{j}$ for all $x \in U_{i}$, since $U_{i j}$ is an atom, and that $\psi\left[U_{j}\right]=U_{j}$ by 2 . above.

2.4. Cylindric algebras. Relation algebras are algebras of binary relations, while cylindric algebras are algebras of relations of higher rank. A cylindric algebra of dimension $\alpha$, where $\alpha$ is a non zero ordinal, is a Boolean algebra $\mathfrak{A}$ with unary additive operations $c_{i}$ and distinguished elements $d_{i j}$ for $i, j<\alpha$, such that for all $i, j, k<\alpha$ and $x \in A$ the following conditions hold:

(1) The $c_{i}$ 's are complemented closure operations commuting with each other, i.e.

(a) $x \leq c_{i} x=c_{i} c_{i} x$

(b) $c_{i}-c_{i} x=-c_{i} x$

(c) $c_{i} c_{j} x=c_{j} c_{i} x$

(2) The constants $d_{i j}$ satisfy the following:

(a) $d_{i j} \cdot d_{j k} \leq d_{i k}, d_{i j}=d_{j i}, d_{i i}=1$

(b) $c_{i} d_{i j}=1, c_{k} d_{i j}=d_{i j}$ if $k \neq i, j$

(c) $\left(c_{i} x\right) \cdot d_{i j}=x$ if $x \leq d_{i j}, i \neq j$.

Note that 2.c expresses that $c_{i}$ is discrete or the identity when relativized to $d_{i j}, i \neq$ $j$. The (equational) class of all cylindric algebras of dimension $\alpha$ is denoted by $C A_{\alpha}$. With some abuse of notation we also denote the elements of $C A_{\alpha}$ by their class, so that we can speak of an algebra $\mathfrak{A}$ being a $C A_{\alpha}$.

A $C A_{\alpha} A$ is called locally finite if $\left\{i<\alpha: c_{i} x \neq x\right\}$ is finite for all $x \in A$. The locally finite $C A_{\alpha}$ s play a crucial role in the algebraization of first order logic in [HMT71], [HMT85].

For a nonzero ordinal $n$ we let $\operatorname{Rel}_{n}(U)$ denote the set of all $n$-ary relations on $U$. If $s \in{ }^{n} U$, we think of $s$ as $\left\langle s_{0}, \ldots, s_{i}, \ldots\right\rangle_{i<n}$. The Boolean operations are 
just the set theoretic ones. The cylindrifications $c_{i}$ can be interpreted as making the information in column $i$ irrelevant, since, after applying $c_{i}$, this column will contain all information. The constants $d_{i j}$ denote the identity relations. Thus, if $R$ is an $n$-ary relation on $U$ and $i, j<n$ then

$$
\begin{gathered}
c_{i} R=\left\{\left\langle s_{0}, \ldots, s_{i-1}, u, s_{i+1}, \ldots\right\rangle: s \in R, u \in U\right\}, \text { and } \\
d_{i j}=\left\{s \in U^{n}: s_{i}=s_{j}\right\} .
\end{gathered}
$$

$\mathfrak{R e l}_{n}(U)$ will denote the full algebra of $n$-ary relations on $U$ with the above operations. By a cylindric set algebra of dimension $n-$ a $C s_{n}$ - on the base set $U$ we mean a subalgebra of $\mathfrak{R e l} l_{n}(U)$, and $\mathbf{I} C s_{n}$ denotes the class of all algebras isomorphic to some $C s_{n}$. A cylindric algebra is called representable if it is isomorphic to a subdirect product of cylindric set algebras.

A binary relation $R \subseteq U \times U$ can always be considered as an $n$-ary relation on $U$, $n \geq 2$, in a natural way: The $n$-ary version $\bar{R}$ of $R$ can be defined as

$$
\bar{R}=\left\{s \in U^{n}:\left\langle s_{0}, s_{1}\right\rangle \in R\right\}
$$

(roughly, $\bar{R}=R \times{ }^{n-2} U$ ). Using this idea, every $C A_{n} \mathfrak{A}$ with $n \geq 3$ has a relation algebraic reduct $\mathfrak{R a} \mathfrak{A}$ defined as follows: For all $i, j<n, i \neq j$ define

$$
s_{j}^{i} x=c_{i}\left(d_{i j} \cdot x\right),
$$

and, using this notation, for all $x, y \in A$ we let

$$
\begin{aligned}
& x \circ y \stackrel{\text { def }}{=} c_{2}\left(s_{2}^{0} x \cdot s_{2}^{1} y\right), \\
& x^{-1} \stackrel{\text { def }}{=} s_{0}^{2} s_{1}^{0} s_{2}^{1} x \\
& 1^{\prime} \stackrel{\text { def }}{=} d_{01} \text {, } \\
& R a \mathfrak{A} \stackrel{\text { def }}{=} \quad\left\{x \in A: c_{i} x=x \text { for all } 2 \leq i<n\right\} .
\end{aligned}
$$

It can be checked that $R a \mathfrak{A}$ is always closed under the Boolean operations as well as under the operations defined above. Now $\mathfrak{R a} \mathfrak{A}$ is the algebra

$$
\mathfrak{R a} \mathfrak{A} \stackrel{\text { def }}{=}\left\langle R a \mathfrak{A},+, \cdot,-, ;,{ }^{\vee}, 0,1,1^{\prime}\right\rangle .
$$

In cylindric set algebras, the operations ${ }^{-1},{ }^{-1}, 1^{\prime}$ mean relation composition, inverse and identity among the binary elements of the algebra.

We call a $C A_{n} \mathfrak{A}$ integral if $\mathfrak{R a} \mathfrak{A}$ is integral; this is equivalent to saying that there are no proper one-dimensional elements in $\mathfrak{A}$, i.e. that

$$
(\forall x \in A)\left[(\forall i, 0<i<n) c_{i} x=x \Rightarrow(x=0 \text { or } x=1)\right] .
$$

The group $A u t_{0}(\mathfrak{A})$ of a $C s_{n} \mathfrak{A}$ with base $U$ is the set of all permutations $\varphi$ of $U$ such that for all $R \in A$

$$
R=\left\{\left\langle\varphi\left(s_{0}\right), \ldots, \varphi\left(s_{i}\right), \ldots\right\rangle: s \in R\right\} .
$$

$\mathfrak{A}$ is called c-permutational if $A u t_{0}(\mathfrak{A})$ is transitive, and a $C A_{n}$ is called permutational if it is isomorphic to a c-permutational one. 


\section{INTEGRAL AND PERMUTATIONAL ALGEBRAS}

It was asked in [McK66], whether every integral RA was permutational. This question was solved in [ADN92] where it was shown that there is an integral representable RA without a permutational representation. If we think of a representable simple RA as an encoding of how the elements of $\mathfrak{A}$ must behave as relations in any representation, then a non - permutational representable RA has a non transitive group of base automorphisms in any of its representations. Thus, the structure of $\mathfrak{A}$ says something about all representations of $\mathfrak{A}$, a somewhat surprising fact if one considers the relationship between relation algebras and $L^{3}$. We note in passing that for $\mathfrak{A} \leq \mathfrak{R e l}(U)$ with $\mathrm{U}$ finite, the property $\mathfrak{A}$ is c - permutational is $\Sigma_{1}^{1}$ expressible with the first order part having just 3 variables: If the atoms of $\mathfrak{A}$ are $R_{0}, \ldots, R_{k-1}$, then $\mathfrak{A}$ is c-permutational if and only if satisfies

$$
(\forall x)(\forall y)(\exists P)\left[P \text { is a permutation } \wedge \bigwedge\left(P \circ R_{i}=R_{i} \circ P\right] .\right.
$$

The first order part can be written with three variables, and the quantified predicate can be moved to the front (with its rank increased), so that the resulting sentence is $\Sigma_{1}^{1}$ expressible. It is not known whether being not permutational can be expressed by a $\Sigma_{1}^{1}$ sentence.

In this section we will explain the logical background of these phenomena. We shall see that the difference between the notions integral and permutational lies in the different expressive powers of the underlying languages.

Let $L$ be any first-order language, $L^{x}$ be the set of $L$-formulas with exactly one free variable, and $\mathfrak{M}=\langle M, \bar{R}\rangle$ be a model of $L$. Here, $\bar{R}$ stands for a sequence of relations of appropriate types. If $a \in M$, then $\langle\mathfrak{M}, a\rangle$ denotes the model $\mathfrak{M}$ expanded with a constant symbol which denotes $a$.

We say that $\mathfrak{M}$ is first-order integral, if no proper subset of $M$ is first-order definable in the model $\mathfrak{M}$, i.e. for all $\varphi \in L^{x}$, the set

$$
\{a \in M: \mathfrak{M}=\varphi[a]\}
$$

is either empty or equal to $M$. We call (the first order model) $\mathfrak{M}$ c-permutational if the group of all automorphisms of $\mathfrak{M}$ is transitive, i.e. if for all $a, b \in M$ there is an automorphism $\sigma$ of $\mathfrak{M}$ with $\sigma(a)=b$. We note that this terminology is in harmony with our previous one: In [HMT85] 4.3.4, to any model $\mathfrak{M}$ a $\mathfrak{C}^{\mathfrak{M}} \in C s_{\omega}$ is associated, namely, $\mathfrak{C}^{\mathfrak{M}}$ is a $C s_{\omega}$ with base $M$ and with universe $\{\operatorname{def} \varphi: \varphi \in$ $L\}$. It is easy to check that $\mathfrak{M}$ is first order - integral iff $\mathfrak{C}^{\mathfrak{M}}$ is integral and $\mathfrak{M}$ is c-permutational iff $\mathfrak{C}^{\mathfrak{M}}$ is c-permutational. We also note that in universal algebra, sometimes the term homogeneous is used instead of c-permutational.

Our first result shows that within the class of locally finite $C s_{\omega} \mathrm{s}$, integral and permutational coincide. As a preparation, we shall prove a model theoretic lemma:

Lemma 3.1. Let $L$ be a first order language and $\mathfrak{M}=\langle M, \bar{R}\rangle$ be a model of $L$. then, the following statements are equivalent:

(1) $\mathfrak{M}$ is first order integral.

(2) $\left(\forall \varphi \in L^{x}\right) \mathfrak{M} \models(\exists x) \varphi \Rightarrow(\forall x) \varphi$.

(3) $(\forall a, b \in M)\langle\mathfrak{M}, a\rangle \equiv\langle\mathfrak{M}, b\rangle$.

(4) $\mathfrak{M}$ has a c-permutational elementary extension. 
Proof. The equivalences $1 . \Leftrightarrow 2 . \Leftrightarrow 3$. and the implication $4 . \Rightarrow 1$. are obvious.

For the rest, suppose that $\mathfrak{M}$ is countable and first order-integral. By [CK71] $3.2 .8, \mathfrak{M}$ has a countably homogeneous elementary extension $\mathfrak{M}^{\prime}$, which is first order-integral by 2 . Then $\mathfrak{M}^{\prime}$ is c-permutational by 3. and by [CK71] 3.2.9.(i). To eliminate the countability restriction, let $\Pi$ be a new fourary relation symbol. Let $\Phi(\Pi)$ be the set of sentences expressing that, for any $x, y$, if we fix $x$ and $y$, then $\Pi(x, y, u, v)$ is an automorphism taking $x$ to $y$ (cf [McK66], p.110.) Now it is straightforward to check that $\mathfrak{M}$ has a c-permutational elementary extension iff $T h(\mathfrak{M}, a)_{a \in M} \cup \Phi(\Pi)$ is consistent (where $T h(\mathfrak{M}, a)_{a \in M}$ denotes the set of formulas valid in $\left.(\mathfrak{M}, a)_{a \in M}\right)$. By the compactness theorem, we may assume that $\mathfrak{M}$ is countable.

Proposition 3.2. Every locally finite $C A_{\omega}$ is permutational.

Proof. Let $\mathfrak{C}$ be an integral locally finite $C A_{\omega}$. Then $\mathfrak{C}$ is simple by [HMT71] 2.3.14, and therefore $\mathfrak{C} \cong \mathfrak{C}^{\mathfrak{M}}$ for some model $\mathfrak{M}$ by [HMT85] 3.2.8 and 4.3.5. By $3.1, \mathfrak{M}$ is elementarily equivalent to some c-permutational $\mathfrak{M}^{\prime}$, and then $\mathfrak{C}^{\mathfrak{M}} \cong$ $\mathfrak{C}^{\mathfrak{M}^{\prime}}$ by [HMT85] 4.3.68(7). Since $\mathfrak{M}^{\prime}$ is c-permutational, $\mathfrak{C}^{\mathfrak{M}^{\prime}}$ is permutational and hence $\mathfrak{C}$ is permutational by $\mathfrak{C} \cong \mathfrak{C}^{\mathfrak{M}^{\prime}}$.

The following example shows that locally finite cannot be omitted from 3.2: Let $U$ be any infinite set, $u, v \in U, u \neq v, R=\{\langle u, u, u, \ldots\rangle\}, S=\{\langle v, v, v, \ldots\rangle\}$, and let $\mathfrak{A}$ be the subalgebra of $\mathfrak{R e l}_{\omega}(U)$ generated by $R$ and $S$. Then $\mathfrak{A}$ is integral by [HMT85] 3.1.59 and [HMT71] 2.1.22. However, $\mathfrak{A}$ is not permutational: Let $\mathfrak{B}$ be any representation of $\mathfrak{A}$, i.e. $\mathfrak{B} \leq \mathfrak{R e l}_{\omega}(W)$ and $h: \mathfrak{A} \rightarrow \mathfrak{B}$ is an isomorphism. Then $h(R) \cup h(S) \subseteq\{\langle w, w, w, \ldots\rangle: w \in W\}, h(R) \neq 0, h(S) \neq 0$, and $h(R) \cap h(S)=0$. Let $w, z \in W$ such that $\langle w, w, w, \ldots\rangle \in h(R),\langle z, z, z, \ldots\rangle \in$ $h(S)$. Then $w \neq z$ and clearly there is no $\varphi \in A u t_{0}(\mathfrak{B})$ such that $\varphi(w)=z$.

Now we turn to relation algebras. We first give a characterisation of the class $\mathcal{P}$ of permutational RA's in terms of cylindric algebras. Roughly, the result says, that a relation algebra is permutational if and only if it can be extended to an integral cylindric algebra of dimension $n$, for arbitrary large $n$.

Proposition 3.3. Let $\mathfrak{A}$ be a relation algebra. Then the following are equivalent:

((i)) $\mathfrak{A}$ is permutational.

((ii)) For all $3 \leq n<\omega, \mathfrak{A} \subseteq \mathfrak{R a} \mathfrak{C}$ for some integral $\mathfrak{C} \in \mathbf{I C s}_{\mathbf{n}}$.

((iii)) For all $3 \leq n<\omega, \mathfrak{A} \subseteq \mathfrak{R a} \mathfrak{C}$ for some integral $\mathfrak{C} \in C A_{n}$.

((iv)) $\mathfrak{A} \subseteq \mathfrak{R a} \mathfrak{C}$ for some integral $\mathfrak{C} \in C A_{\omega}$.

Proof. $(i) \Rightarrow(i i)$ : Assume, $\mathfrak{A}$ is a permutational relation algebra, say, $\mathfrak{A} \cong \mathfrak{A}^{\prime}$ where $\mathfrak{A}^{\prime} \leq \mathfrak{R e l}(U)$ for some $U$ and $\mathfrak{A}^{\prime}$ is c-permutational. Let $3 \leq n<\omega$, and let $\mathfrak{C}$ be the subalgebra of $\mathfrak{R e l}_{n}(U)$ generated by $\left\{\bar{R}: R \in A^{\prime}\right\}$. Then $\overline{\mathfrak{A}}^{\prime}$ is isomorphic to a subalgebra of $\mathfrak{R a} \mathfrak{C}$. By [HMT85] 3.1.36 we have $A u t_{0}(\mathfrak{C})=A u t_{0}\left(\mathfrak{A}^{\prime}\right)$, hence $\mathfrak{C}$ is permutational, and thus it is integral.

(ii) $\Rightarrow$ (iii) : Obvious, since $\mathbf{I} C s_{n} \subseteq C A_{n}$.

(iii) $\Rightarrow\left(\right.$ iv) : Suppose that $\mathfrak{A} \subseteq \mathfrak{R a} \mathfrak{C}_{n}$ where $\mathfrak{C}_{n}$ is an integral $C A_{n}$ for each $3 \leq n<\omega$. For each such $n$ let $\mathfrak{C}_{n}^{\prime}$ be an arbitrary expansion of $\mathfrak{C}_{n}$ such that $\mathfrak{C}_{n}^{\prime}$ is an algebra similar to $C A_{\omega}$ 's. Let $F$ be any nonprincipal ultrafilter on $N=\{n<$ $\omega: n \geq 3\}$ and let $\mathfrak{C}=\prod\left\langle\mathfrak{C}_{n}^{\prime} \mid n \in N\right\rangle / F$ be the ultraproduct of the algebras $\mathfrak{C}_{n}^{\prime}$ 
with respect to $F$. Now $\mathfrak{C} \in C A_{\omega}$ and $h: \mathfrak{A} \longmapsto \mathfrak{R a} \mathfrak{C}$ is an embedding, where $h(a)=\langle a, a, a, \ldots\rangle / F$ for all $a \in A$. We will show that the subalgebra $\mathfrak{C}^{\prime}$ of $\mathfrak{C}$ generated by the range of $h$ is integral. This will imply that $\mathfrak{A} \subseteq \mathfrak{R a} \mathfrak{C}^{\prime \prime}$ for some integral $\mathfrak{C}^{\prime \prime} \in C A_{\omega}$.

Thus, let $x \in C^{\prime}$ be arbitrary. Then $x=\tau\left(h\left(a_{1}\right), \ldots, h\left(a_{k}\right)\right)$ for some $C A_{\omega}$-term $\tau$ and for some $a_{1}, \ldots, a_{k} \in A$. Let $m \geq 3$ be an upper bound of the indices occurring in $\tau$. Then $\tau$ is a $C A_{n}$-term for all $n \geq m$ and $x=\left\langle x_{n}: n \in N\right\rangle / F$ where $x_{n}=\tau^{\mathfrak{C}_{n}}\left(a_{1}, \ldots, a_{k}\right)$ for all $n \geq m$.

Assume $x=c_{i} x$ in $\mathfrak{C}^{\prime}$ for all $0<i<\omega$, i.e. that $x$ is an at most one-dimensional element of $\mathfrak{C}^{\prime}$. Then, there is some $m_{1} \geq m, m_{1} \in N$, such that $x_{n}=c_{i} x_{n}$ in $\mathfrak{C}_{n}^{\prime}$ for all $n \geq m_{1}$ and all $0<i \leq m$. Let $n \geq m_{1}$; then, $x_{n}=c_{i} x_{n}$ in $\mathfrak{C}_{n}^{\prime \prime}$ for all $0<i<n$ : This follows from the facts that for $m<i<n, i$ does not occur in $\tau$ by our choice of $m$, and that $c_{i} a_{j}=a_{j}$ for all $1 \leq j \leq k$. The integrality of $\mathfrak{C}_{n}$ now implies that $x_{n} \in\{0,1\}$. Since this is true for all $n \geq m_{1}-$ hence, for cofinitely many elements of $N$ - we have $x \in\{0,1\}$. Thus $\mathfrak{C}^{\prime}$ is integral.

$(i v) \Rightarrow(i)$ : Assume $\mathfrak{A} \subseteq \mathfrak{R a} \mathfrak{C}$ for some integral $\mathfrak{C} \in C A_{\omega}$, and let $\mathfrak{C}^{\prime}$ be the subalgebra of $\mathfrak{C}$ generated by $A$. Then, $\mathfrak{C}^{\prime}$ is an integral locally finite $C A_{\omega}$, thus $\mathfrak{C}^{\prime}$ is permutational by 3.2. This easily implies that $\mathfrak{R a} \mathfrak{C}^{\prime}$ is permutational, and therefore $\mathfrak{A} \subseteq \mathfrak{R a} \mathfrak{C}^{\prime}$ is permutational.

Any $\mathfrak{A} \leq \mathfrak{R e l}(U)$ can be considered a first order model $\mathfrak{M}(\mathfrak{A})$, namely $\mathfrak{M}(\mathfrak{A})=$ $\langle U, a\rangle_{a \in A}$. The universe of this model $\mathfrak{M}(\mathfrak{A})$ is $U$ and all the relations of $\mathfrak{M}(\mathfrak{A})$ are binary. From 2.3.1 we know that what we can express by RA-terms in $\mathfrak{A}$ is the same as what we can express in $\mathfrak{M}(\mathfrak{A})$ by first-order formulas using only 3 variables. From this we obtain

(1) $\mathfrak{A}$ is integral iff no proper nonempty subset of $U$ can be defined over the model $\mathfrak{M}(\mathfrak{A})$ by formulas using only 3 variables.

(2) $\mathfrak{A}$ is c-permutational iff $\mathfrak{M}(\mathfrak{A})$ is c-permutational.

It is easy to see that for an integral $\mathfrak{A} \leq \mathfrak{R e l}(U), \mathfrak{M}(\mathfrak{A})$ is not necessarily firstorder integral (see [ADN95]): Let $S={ }^{2} \mathbf{3} \cup{ }^{2}(\mathbf{7} \backslash \mathbf{3}) . S$ is the disjoint union of a $\mathrm{K}_{3}$ and a $\mathrm{K}_{4}$, and it generates an integral relation algebra $\mathfrak{A} \leq \mathfrak{R e l}(\boldsymbol{7})$ with atoms $S, T, 1^{\prime}$ and the following relative composition table:

\begin{tabular}{|c||c|c|}
\hline$\circ$ & $S$ & $T$ \\
\hline \hline$S$ & $-T$ & $T$ \\
\hline$T$ & $T$ & $-T$ \\
\hline
\end{tabular}

By the integrality of $\mathfrak{A}$, no proper nonempty subset of $\mathbf{7}$ can be defined with three variables, but $\{3,4,5,6\}$ can be defined with four variables. Hence, $\mathfrak{M}(\mathfrak{A})$ is not first-order integral.

We now turn to the logical background of the difference between the notions integral and permutational. It will turn out that this difference is rooted in the fact that 3 -variable logic is strictly weaker than full first order logic.

As in [McK66], p. 108, the representation language $L^{\mathfrak{A}}$ of some $\mathfrak{A} \in R A$ is defined as follows: $L^{\mathfrak{A}}$ is a first-order language with equality and binary relation symbols $\left\{R_{a}: a \in A\right\} . L^{\mathfrak{A}, x}$ is the set of formulas of $L^{\mathfrak{A}}$ with just one free variable $x$, and $L_{k}^{\mathfrak{A}, x}$ the set of formulas of $L^{\mathfrak{A}, x}$ containing at most $k$ distinct variables 
(cf. [HMT85], 4.3). The set $T h_{r}(\mathfrak{A})$ of sentences of $L^{\mathfrak{A}}$ which describe $\mathfrak{A}$ is the following:

$$
\begin{aligned}
T h_{r}(\mathfrak{A})= & \left\{(\exists x \exists y) R_{a}(x, y): a \in A, a \neq 0\right\} \cup \\
& \left\{( \forall x \forall y ) \left(\left[R_{a^{-}}(x, y) \leftrightarrow \neg R_{a}(x, y)\right] \wedge\right.\right. \\
& {\left[R_{a+b}(x, y) \leftrightarrow\left(R_{a}(x, y) \vee R_{b}(x, y)\right)\right] \wedge } \\
& {\left[R_{a ; b}(x, y) \leftrightarrow(\exists z)\left(R_{a}(x, z) \wedge R_{b}(z, y)\right)\right] \wedge } \\
& {\left[R_{a^{\vee}}(x, y) \leftrightarrow R_{a}(y, x)\right] \wedge } \\
& {\left.\left.\left[R_{1^{\prime}}(x, y) \leftrightarrow x=y\right]\right): a, b \in A\right\} . }
\end{aligned}
$$

If $\mathfrak{A}$ is simple, there is a one-one correspondence between representations of $\mathfrak{A}$ and models of $T h_{r}(\mathfrak{A})$ : Suppose that $\mathfrak{B} \leq \mathfrak{R e l}(U)$ and $f: \mathfrak{A} \rightarrow \mathfrak{B}$ is an isomorphism. Define $\mathfrak{M}=\mathfrak{M}(\mathfrak{B}, f)=\langle U, f(a)\rangle_{a \in A}$, i.e. in $\mathfrak{M}$ the relation symbol $R_{a}$ denotes $f(a) \subseteq U \times U$ for all $a \in A$. Then $\mathfrak{M}$ is a model of the language $L^{\mathfrak{A}}$, and $\mathfrak{M}=T h_{r}(\mathfrak{A})$.

Conversely, if $\mathfrak{N}=\left\langle N, R_{a}^{\mathfrak{N}}\right\rangle_{a \in A}$ is any model of $L^{\mathfrak{A}}$ with $\mathfrak{N} \models T h_{r}(\mathfrak{A})$, the function $f$ defined by $f(a)=R_{a}^{\mathfrak{N}}$ for all $a \in A$ defines a representation of $\mathfrak{A}$, i.e. $f$ is an isomorphism from $\mathfrak{A}$ onto a BRA over $N$. In particular, $\mathfrak{A}$ is representable iff $T h_{r}(\mathfrak{A})$ is consistent.

Proposition 3.4. Let $\mathfrak{A}$ be a simple and representable relation algebra. Then,

(1) $\mathfrak{A}$ is integral iff $\quad\left(\forall \varphi \in L_{3}^{\mathfrak{A}, x}\right) T h_{r}(\mathfrak{A}) \models(\exists x) \varphi \rightarrow(\forall x) \varphi$.

(2) $\mathfrak{A}$ is permutational iff $\quad \operatorname{Th}_{r}\left(\mathfrak{A} \cup\left\{(\exists x) \varphi \rightarrow(\forall x) \varphi: \varphi \in L^{\mathfrak{A}, x}\right\}\right.$ is consistent.

Proof. 1. We have seen that there is a one-one correspondence between representations of $\mathfrak{A}$ and between models of $T h_{r}(\mathfrak{A})$. It is proved in [TG87] 3.9 and in [HMT85] 5.3.12, that in this correspondence, relation algebraic terms correspond exactly to formulas using three variables two of which are free. More precisely, suppose that $\mathfrak{A} \leq \mathfrak{R e l}(U)$ and that $\mathfrak{M}=(U, a)_{a \in A}$. Then

(1) For all RA-terms $\tau$ there is some $\varphi \in \mathcal{L}_{3}^{\mathfrak{A}}$ with at most two free variables such that

$$
(\star) \quad \tau^{\mathfrak{A}}=\{(u, v) \in U \times U: \mathfrak{M}=\varphi(u, v)\} .
$$

(2) Conversely, for all $\varphi \in \mathcal{L}_{3}^{\mathfrak{A}}$ with at most two free variables there is an RA-term $\tau$ such that $(\star)$ holds.

Now $\mathfrak{A}$ is integral iff for all RA-terms $\tau$ we have $\tau \circ 1 \in\{0,1\}$ by 2.3.2 (3). By 1. and 2. above this then holds iff $\mathfrak{M} \not \forall(\exists x \varphi \wedge \exists x \neg \varphi)$ for all $\varphi \in \mathcal{L}_{3}^{\mathfrak{A}, x}$, i.e. if $\mathfrak{M}=\exists x \varphi \rightarrow \forall x \varphi$ for all $\varphi \in \mathcal{L}_{3}^{\mathfrak{A}, x}$. By $\mathfrak{M} \mid=T h_{r}(\mathfrak{A})$ we are done.

2. " $\Rightarrow$ ": Suppose that $\mathfrak{A}$ is permutational, $\mathfrak{B}$ is c-permutational and isomorphic to $\mathfrak{A}$, and that $\mathfrak{M} \stackrel{\text { def }}{=} \mathfrak{M}(\mathfrak{B})$. Then $\mathfrak{M}=T h_{r}(\mathfrak{A})$, and every $\sigma \in A u t_{0}(\mathfrak{B})$ is an automorphism of $\mathfrak{M}$. Assume that $u, v \in U$ with $\mathfrak{M} \models \varphi(u)$ and $\mathfrak{M} \models \neg \varphi(v)$. Since $\mathfrak{B}$ is c-permutational, there is some $\sigma \in A u t_{0}(\mathfrak{B})$ such that $\sigma(u)=v$. Therefore, $\mathfrak{M} \models \varphi(u)$ implies $\mathfrak{M} \models \varphi(\sigma(u))$, since $\sigma$ is an automorphism of $\mathfrak{M}$, a contradiction. Hence, $\mathfrak{M} \models(\exists x) \varphi \rightarrow(\forall x) \varphi$ for all $\varphi \in L$ as well

"६": Let $\mathfrak{M}=\left\langle U, R_{a}^{\mathfrak{M}}\right\rangle_{a \in A}$ be a model of

$$
T h_{r}(\mathfrak{A}) \cup\left\{(\exists x) \varphi \rightarrow(\forall x) \varphi: \varphi \in L_{3}^{\mathfrak{A}, x}\right\} .
$$


Then no proper nonempty subset of $\mathfrak{M}$ is first-order definable, and hence we may assume by Lemma 3.1 that $\mathfrak{M}$ is c-permutational. But then $\left.\left\{R_{a}^{\mathfrak{M}}: a \in A\right\rangle\right\}$ is a c-permutational representation of $\mathfrak{A}$, which shows that $\mathfrak{A}$ is permutational.

We see from this result that being permutational is a general first property for $\mathfrak{M}(\mathfrak{A})$. In the language of relation algebras, being not permutational is not general first order: In [ADN92] we have constructed an infinite family of non permutational finitely representable relation algebras an ultraproduct of which is permutational.

We can state the analogous theorem for algebras of $n$-ary relations. If $\mathfrak{A} \in$ $C A_{n}, n<\omega$, then the representation language $\mathcal{L}^{\mathfrak{A}}$ of $\mathfrak{A}$ has an $n$-ary relation symbol $R_{a}$ for all $a \in A$, and $T h_{r}(\mathfrak{A})$ is defined as

$$
\begin{aligned}
T h_{r}(\mathfrak{A})= & \left\{(\exists \bar{x}) R_{a}(\bar{x}): a \in A, a \neq 0\right\} \cup \\
& \left\{( \forall \overline { x } ) \left(\left[R_{a^{-}}(\bar{x}) \leftrightarrow \neg R_{a}(\bar{x})\right] \wedge\right.\right. \\
& {\left[R_{a+b}(\bar{x}) \leftrightarrow\left(R_{a}(\bar{x}) \vee R_{b}(\bar{x})\right) \wedge\right.} \\
& {\left[R_{c_{i} a}(\bar{x}) \leftrightarrow\left(\exists x_{i}\right)\left(R_{a}(\bar{x})\right)\right] \wedge } \\
& {\left.\left.\left[R_{d_{i j}}(\bar{x}) \leftrightarrow x_{i}=x_{j}\right]\right): a, b \in A, i, j<n\right\} . }
\end{aligned}
$$

Clearly, a simple $\mathfrak{A} \in C A_{n}$ is representable iff $T h_{r}(\mathfrak{A})$ is consistent, and there is a one-one correspondence between representations of $\mathfrak{A}$ and models of $T h_{r}(\mathfrak{A})$.

Proposition 3.5. Let $3 \leq n<\omega$ and let $\mathfrak{A} \in C A_{n}$ be simple and representable. Then,

(1) $\mathfrak{A}$ is integral $\quad$ iff $\quad\left(\forall \varphi \in L_{3}^{\mathfrak{A}, x}\right) T h_{r}(\mathfrak{A}) \models(\exists x) \varphi \rightarrow(\forall x) \varphi$.

(2) $\mathfrak{A}$ is permutational iff $\quad T h_{r}\left(\mathfrak{A} \cup\left\{(\exists x) \varphi \rightarrow(\forall x) \varphi: \varphi \in L^{\mathfrak{A}, x}\right\}\right.$ is consistent.

Since the proof of 3.5 is completely analogous to that of 3.4 we leave it to the reader. Similar theorems can be stated for various algebraizations of logic, e.g. for polyadic algebras.

3.4 has the following consequence for BRAs:

Corollary 3.6. (1) For BRAs, the property "integral" is expressible by a $L_{\omega_{1} \omega}^{3}$ formula.

(2) For BRAs, the property "c-permutational" is not expressible by a $L_{\omega_{1} \omega}^{\omega}$ formula.

Proof. 1. Let $\mathfrak{A}$ be a BRA, and enumerate all formulas of $L_{3}^{\mathfrak{A}, x}$ by $\left\{\varphi_{n}: n<\omega\right\}$. then, by 3.4 ,

$$
\mathfrak{A} \text { is integral iff } \mathfrak{M}(\mathfrak{A}) \models \bigwedge_{n<\omega}\left[(\exists x) \varphi_{n} \rightarrow(\forall x) \varphi_{n}\right] .
$$

2. We shall show that for each $n<\omega$ there are BRAs $\mathfrak{A}$ and $\mathfrak{B}$ such that

(1) $\mathfrak{A}$ is c-permutational,

(2) $\mathfrak{B}$ is not c-permutational,

(3) $\mathfrak{M}(\mathfrak{A})$ and $\mathfrak{M}(\mathfrak{B})$ satisfy the same $L_{\omega_{1} \omega}^{n}$ sentences.

Let

(1) $|U|=2(n+1), R \subseteq U \times U$ be a disjoint union of two $\mathrm{K}_{n+1} \mathrm{~s}$, and $\mathfrak{A}=\langle R\rangle$,

(2) $|V|=2 n+1, S \subseteq V \times V$ be a disjoint union of a $K_{n}$ and a $\mathrm{K}_{n+1}$, and $\mathfrak{B}=\langle S\rangle$. 
Then, $\mathfrak{A}$ is permutational, while $\mathfrak{B}$ is not. To show 3 ., we first observe that it suffices to consider the structures $\langle U, R\rangle$ and $\langle V, S\rangle$, since $\mathfrak{A}$ and $\mathfrak{B}$ are one - generated by $R$, resp. $S$. Now we play the $n$-pebble game of [KV92] 2.14. First note that a $\mathrm{K}_{n}$ and a $\mathrm{K}_{n+1}$ cannot be distinguished in $L_{\omega_{1} \omega}^{n}$ : Partial isomorphisms between these structures depend only on the number of pebbles played. Since this number is never greater than $n$, the duplicator can at any stage match a move made by the spoiler. Now, the duplicator fixes a correspondence between the components of $R$ and the components of $S$, and matches any move made by the spoiler by the corresponding component in the other structure. Since the duplicator wins the game on the components, the spoiler can never win a round of the game.

\section{RIGID RELATION ALGEBRAS}

In the previous section we have explored permutational algebras, i.e. those with a representation having a transitive group of base automorphisms. We have seen that $\mathfrak{A} \leq \mathfrak{R e l}(U)$ is c - permutational if no proper nonempty subset of $U$ is definable in $\mathfrak{M}(\mathfrak{A})$. At the other end of the spectrum are the rigid algebras: Let $\mathfrak{A} \leq \mathfrak{R e l}(U)$. $\mathfrak{A}$ is called $c$ - rigid if $\mathfrak{A}^{\rho}=1^{\prime}$, i.e. if $\mathfrak{A}$ has no proper base automorphisms. A representable $\mathfrak{A}$ is called rigid if it has a c - rigid representation. Finally, we call $R \in \operatorname{Rel}(U)$ rigid if the only permutation of $U$ which preserves $R$ is the identity.

Throughout this section we will suppose that $U$ is a finite set, and that all RAs mentioned are finitely representable, i.e. for each such $\mathfrak{A}$ there is a finite set $U$ and some $\mathfrak{B} \leq \mathfrak{R e l}(U)$ such that $\mathfrak{A} \cong \mathfrak{B}$.

Proposition 4.1. (1) $\mathfrak{A} \leq \mathfrak{R e l}(U)$ is c-rigid if and only if $\mathfrak{A}^{\rho \sigma}=\mathfrak{R e l}(U)$.

(2) If $\mathfrak{A}$ is a maximal proper subalgebra of $\mathfrak{R e l}(U)$, then $\mathfrak{A}$ is either c-rigid or Galois closed.

Proof. 1. " $\Rightarrow ":$ Since $\mathfrak{A}$ is c-rigid, $\mathfrak{A}^{\rho}=\left\{1^{\prime}\right\}$, and it follows that $\mathfrak{A}^{\rho \sigma}=\mathfrak{R e l}(U)$. $" \Leftarrow "$ : Suppose that $\mathfrak{A}$ is not c-rigid. Then, there are some $\varphi \in \mathfrak{A}^{\rho}$ and $a, b \in U$ such that $a \neq b$ and $\varphi(a)=b$. The atom $\mathfrak{A}^{\rho}(a, a)$ of $\mathfrak{A}^{\rho \sigma}$ contains $\langle a, a\rangle$ as well as $\langle b, b\rangle$. On the other hand, every atom of $\mathfrak{R e l}(U)$ contains only one element, and thus $\mathfrak{A}^{\rho \sigma} \subsetneq \operatorname{Rel}(U)$.

2. If $\mathfrak{A}$ is not Galois closed, then $\mathfrak{A}$ is a proper subalgebra of $\mathfrak{A}^{\rho \sigma}$. The maximality of $\mathfrak{A}$ implies that $\mathfrak{A}^{\rho \sigma}=\operatorname{Rel}(U)$, and thus $\mathfrak{A}$ is c-rigid by 1 .

The following was shown in [ADN95]:

Proposition 4.2. Let $\mathfrak{A} \leq \mathfrak{R e l}(U)$ and $D \subseteq U$ be nonempty. Then, $D$ is definable in $\mathfrak{M}(\mathfrak{A})$ if and only if $D$ is a union of orbits of $\mathfrak{A}^{\rho}$.

Corollary 4.3. $\mathfrak{A} \leq \mathfrak{R e l}(U)$ is c-rigid if and only if every subset (and hence every relation) of $U$ is definable in $\mathfrak{M}(\mathfrak{A})$.

Proof. If $\mathfrak{A}$ is c-rigid, then each singleton $\{a\}$ is an orbit of $\mathfrak{A}^{\rho}$. Conversely, if every subset of $U$ is definable, then the orbits of $\mathfrak{A}^{\rho}$ are the singletons $\{a\}, a \in U$, and it follows that $\mathfrak{A}^{\rho}=\left\{1^{\prime}\right\}$.

More concretely,

Lemma 4.4. Suppose that $U=\{0, \ldots, n-1\}$, and that $R$ is a rigid relation on $U$. Then, for every $k \in U$, there exists a prenex existential formula which defines $k$ in the model $\langle U, R\rangle$. 
Proof. For all $i, j<n$ let

$$
\varphi_{i j} \stackrel{\text { def }}{=} \begin{cases}v_{i} R v_{j}, & \text { if }\langle i, j\rangle \in R \\ \neg v_{i} R v_{j}, & \text { otherwise. }\end{cases}
$$

Furthermore, let $\psi_{i j}$ be the formula $v_{i} \neq v_{j}$. For each $k<n$ let

$$
\chi_{k}=\left(\exists v_{0}\right) \ldots\left(\exists v_{k-1}\right)\left(\exists v_{k+1}\right) \ldots\left(\exists v_{n-1}\right)\left(\bigwedge_{i, j<n} \varphi_{i j} \wedge \bigwedge_{i<j<n} \psi_{i j}\right) .
$$

Clearly, $k \in \operatorname{def} \chi_{k}$. Assume that $m \in \operatorname{def} \chi_{k}$, where $m \neq k$. Let $x_{0}, \ldots, x_{k-1}, x_{k+1}, \ldots, x_{n-1}$ be the elements of $U$ whose existence is asserted by $\chi_{k}$, and set $x_{k} \stackrel{\text { def }}{=} m$. Then, for all $i, j<n$, we have

$$
x_{i} R x_{j} \Longleftrightarrow i R j,
$$

and the $x_{i}$ are pairwise distinct. Hence, the mapping which carries $x_{i}$ to $i$ for each $i<n$ is a base automorphism of $R$ taking $k$ to $m$. Since $k \neq m$, this contradicts the rigidity of $R$.

4.2 implies that $\mathfrak{A} \leq \mathfrak{R e l}(U)$ is c-rigid iff there is some $k<\omega$ such that the $\mathrm{Cs}_{k}$ with base $U$ generated by the canonical embedding of $\mathfrak{A}$ into $\mathfrak{R e l}_{k}(U)$ is the full $k$-dimensional cylindric algebra with base $U$.

The formula given in 4.4 uses $|U|$ variables, and one wonders whether this can be reduced. Let us call $R \in \operatorname{Rel}(U)$ (resp. $\mathfrak{A} \leq \mathfrak{R e l}(U)) k$-rigid if every subset of $U$ is the definable over $\langle U, R\rangle$ (resp. $\mathfrak{M}(\mathfrak{A})$ ) by a first order formula with at most $k$ variables.

B. Jónsson has raised the question whether every rigid relation on $U$ generates $\mathfrak{R e l}(U)$, in other words, whether every rigid relation is 3-rigid. We observe that a positive answer would imply that the property rigid is in the complexity class $\mathbf{P}$ : For a given $R$, find the atoms of $\mathfrak{A} \stackrel{\text { def }}{=}<R>$ in at most $O\left(n^{6}\right)$ steps, and then test in at most $n^{2}$ steps whether $\langle a, a\rangle \in A t(\mathfrak{A})$ for every $a \in U$.

The problem was solved by Andréka and Maddux (and independently by R. McKenzie) who exhibited a symmetric rigid relation on a set with 11 elements which generates an integral BRA with three atoms. Here we give such a relation on the set $U=\{0, \ldots, 9\}$ :

$$
\begin{aligned}
& 0 \rightarrow 4579, \quad 1 \rightarrow 2679 \\
& 2 \rightarrow 13478, \quad 3 \rightarrow 24568 \\
& 4 \rightarrow 02359, \quad 5 \rightarrow 0347 \\
& 6 \rightarrow 1389, \quad 7 \rightarrow 0125 \\
& 8 \rightarrow 2369, \quad 9 \rightarrow 01468
\end{aligned}
$$

The logical background of Jónsson's question is best explained by using the finite variable fragment of $L_{\omega_{1} \omega}$ :

Proposition 4.5. (1) The property " $\mathfrak{A}$ is $k$-rigid" is expressible by a formula of $L_{\omega_{1} \omega}^{k}$.

(2) The property "'A is rigid" is not expressible by a formula of $L_{\omega_{1} \omega}^{\omega}$. 
Proof. 1. Let $\left\{\varphi_{n}: n<\omega\right\}$ be the set of all first order formulas of $L^{\mathfrak{A}}$ with $k$ variables, exactly one of which is free. Then, $\mathfrak{M}(\mathfrak{A})$ is $k$-rigid if and only if

$$
(\forall x)(\forall y)\left[x \neq y \Rightarrow \sum_{n=0}^{\infty}\left(\varphi_{n}(x) \wedge \neg \varphi_{n}(y)\right] .\right.
$$

2. This was shown in [BH79], see also [Com88] for an explanation of the technique; below, we shall give a more direct proof.

The previous result implies that for arbitrarily large $k<\omega$ there is a c-rigid RA which is not $k$-rigid. The original proof of this is probabilistic, using the countable random graph. In [BH79] it is stated that A more technical problem is to find graphs that satisfy many of our axioms. The axioms mentioned are the extension axioms $T_{n}$, which roughly state that for a binary relation $R$, the model $\langle U, R\rangle$ satisfies $T_{n}$ iff for any $2 n$ points of $U$ there is some $z \in U$ connected to the first $n$ vertices but not to the other $n$. We shall now exhibit for each $n \in \omega$ a rigid symmetric (binary) relation $R_{n}$ on a set $U_{n}$ which satisfies all axioms $T_{i}$ for $i \leq n$, and then proceed to show that not all relations can be defined in the model $\left\langle U_{n}, R_{n}\right\rangle$ by formulas with $\mathrm{n}$ variables. As a preparation we shall prove two combinatorial lemmas.

Lemma 4.6. For any $n, m \in \omega$ there are a finite set $S$ and a function $f:{ }^{n} S \rightarrow S$ such that

((i)) $s \prec z \Rightarrow z \nprec s$ for all $s, z \in S$.

((ii)) $\frac{\left|C\left(z_{1}\right) \cap C\left(z_{2}\right)\right|}{|S|}>1-\frac{1}{m}$ for all $z_{1}, z_{2} \in S$.

Here,

$$
\begin{array}{rll}
s \prec z & \stackrel{\text { def }}{\Longleftrightarrow} \quad\left(\exists_{1}, \ldots, s_{n} \in S\right)(\exists i \leq n)\left(s=s_{i} \wedge f\left(s_{1}, \ldots, s_{n}\right)=z\right), \\
C(z) & \stackrel{\text { def }}{=} & \{s \in S: \text { Neither } s \prec z \text { nor } z \prec s\} .
\end{array}
$$

Proof. Let $H$ be a set with $|H|=k>n \cdot(n+1)$, and

$$
S \stackrel{\text { def }}{=}\{\langle K, u\rangle: K \subseteq H,|K|=n+1, u \in K\} .
$$

We define $f:{ }^{n} S \rightarrow S$ as follows: Let $\left\langle S_{1}, u_{1}\right\rangle, \ldots,\left\langle S_{n}, u_{n}\right\rangle \in S$; choose some $L \subseteq H$ and some $w \in H$ such that

(1) $w \notin K_{1} \cup \ldots \cup K_{n}$ (which exists by our assumption on the cardinality of $H)$,

(2) $\left\{u_{1}, \ldots, u_{n}, w\right\} \subseteq L$,

(3) $|L|=n+1$.

Now, set

$$
f\left(\left\langle K_{1}, u_{1}\right\rangle, \ldots,\left\langle K_{n}, u_{n}\right\rangle\right) \stackrel{\text { def }}{=}\langle L, w\rangle .
$$

Suppose that $\left\langle K_{1}, u_{1}\right\rangle \prec\left\langle K_{2}, u_{2}\right\rangle$. Then, there are $\left\langle T_{1}, v_{1}\right\rangle, \ldots,\left\langle T_{n}, v_{n}\right\rangle \in S$ and some $1 \leq i \leq n$ such that $\left\langle K_{1}, u_{1}\right\rangle=\left\langle T_{i}, v_{i}\right\rangle$ and

$$
\begin{aligned}
f\left(\left\langle T_{1}, v_{1}\right\rangle, \ldots\left\langle T_{n}, v_{n}\right\rangle\right) & =\langle L, w\rangle \\
& =\left\langle K_{2}, u_{2}\right\rangle,
\end{aligned}
$$

where

(1) $w \notin T_{1} \cup \ldots \cup T_{i-1} \cup K_{1} \cup T_{i+1} \cup \ldots \cup T_{n}$,

(2) $L \supseteq\left\{v_{1}, \ldots, v_{i-1}, u_{1}, v_{i+1}, \ldots, v_{n}, w\right\}$,

(3) $|L|=n+1$. 
Thus, $u_{1} \in K_{2}, u_{2} \notin K_{1}$, and it follows that (i) holds. We also note that $u_{1} \in$ $K_{1} \cap K_{2}$.

To show (ii) let $s=\left\langle K_{1}, u_{1}\right\rangle, z=\left\langle K_{2}, u_{2}\right\rangle$, and set

$$
M \stackrel{\text { def }}{=}\left\{\langle K, u\rangle: K \subseteq H \backslash\left(K_{1} \cup K_{2}\right),|K|=n+1, u \in K\right\} .
$$

Let $t=\langle K, u\rangle \in M$; then, $u_{1} \notin K$ and $u \notin K_{1}$, since $u \in K, u_{1} \in K_{1}$, and $K \cap K_{1}=\emptyset$. It follows that neither $s \prec t$ nor $t \prec s$, and hence $M \subseteq C(s)$; similarily, $M \subseteq C(z)$.

If $L$ is a set with $|L|=\lambda$ and $\sigma(L) \stackrel{\text { def }}{=}\{\langle K, u\rangle: K \subseteq L,|K|=n+1, u \in K\}$, then

$$
|\sigma(L)|=\frac{\lambda \cdot(\lambda-1) \cdot \ldots \cdot(\lambda-n)}{n !} \cdot(n+1) .
$$

Thus,

$$
\begin{aligned}
\frac{|C(s) \cap C(z)|}{|S|} & >\frac{\left|\sigma\left(H \backslash\left(K_{1} \cup K_{2}\right)\right)\right|}{|\sigma(H)|} \\
& =\frac{(k-2 n-2) \cdot \ldots \cdot(k-3 n-2)}{k \cdot \ldots \cdot(k-n)} \\
& >\left(\frac{k-3 n-2}{k-n}\right)^{n+1},
\end{aligned}
$$

which approaches 1 as $k$ approaches infinity. Thus, given $m$, we can choose $H$ large enough so that (ii) will hold for the corresponding $S$.

Lemma 4.7. Let $k, n \in \omega$. There are disjoint sets $H, M$ and a relation $R \subseteq H \times M$ such that

(1) $\frac{|H|}{|K|}>k$, and

(2) For all $K \subseteq H$ with $|K|=n$ and all $L \subseteq K$ there is a $p \in M$ such that for all $u \in K$,

$$
u R p \Longleftrightarrow u \in L .
$$

Proof. Let $m \in \omega$, and $H$ be the set of all 0-1 sequences of length $m$. Now, set

$$
M \stackrel{\text { def }}{=}\left\{\langle P, Q\rangle: P \subseteq m,|P|=n^{2}, Q \subseteq{ }^{P} 2\right\},
$$

and define $R \subseteq H \times M$ by

$$
s R\langle P, Q\rangle \stackrel{\text { def }}{\Longleftrightarrow} s \uparrow P \in Q .
$$

Here, $s \uparrow P$ is the restriction of the function $s$ to $P$.

Suppose that $m>n^{2}$, and let $|K|=n, K=\left\{s_{1}, \ldots, s_{n}\right\} \subseteq H$, and $L \subseteq K$. Since all $s_{i} \in K$ are different, it follows that for all $\leq i, j \leq n, i \neq j$, there is some $\mu(i, j) \in m$ such that $s_{i}(\mu(i, j)) \neq s_{j}(\mu(i, j))$. Thus, there is some set $\{\mu(i, j): 1 \leq i, j \leq n, i \neq j\} \subseteq P$ with $|P|=n^{2}$ and $s_{i}\left|P \neq s_{j}\right| P$ for all $1 \leq i, j \leq n, i \neq j$.

Let $Q \stackrel{\text { def }}{=}\left\{s_{i}\left\lceil P: s_{i} \in L\right\}\right.$. Then, for all $s \in K$,

$$
s R\langle P, Q\rangle \Longleftrightarrow s \mid P \in Q \Longleftrightarrow s \in L .
$$


Let $\kappa \stackrel{\text { def }}{=}|H|\left(=2^{m}\right)$. Then, $m=\log _{2} \kappa$, and

$$
|M|=m \cdot(m-1) \cdot \ldots \cdot\left(m-n^{2}-1\right) \cdot \frac{1}{\left(n^{2}\right) !} \cdot 2^{2^{\left(n^{2}\right)}} .
$$

If $n$ is fixed, then it is easy to see that for any positive constant $c$,

$$
\lim _{k \rightarrow \infty}\left(\frac{k}{\log _{2} k \cdot \ldots \cdot \log _{2}\left(k-n^{2}-1\right)} \cdot c\right)=1 .
$$

Thus, if $m$ increases, $|M|$ becomes arbitrarily small relative to $|H|$, so that eventually we have $\frac{|H|}{|M|}>k$.

Proposition 4.8. For any $n \in \omega$ there is a symmetric and irreflexive rigid relation on a finite set $U$ such that for all $K \subseteq U$ with $|K| \leq n$ and for all $H \subseteq K$ there is some $p \in U$ such that for all $u \in K$,

$$
u R p \Longleftrightarrow u \in H .
$$

Proof. Fix some $n \in \omega$, and let $S$ and $f$ be as in 4.6 with $m=4$ and $|S| \geq 5$. Furthermore, let $H^{\prime}, M, R^{\prime}$ be as in 4.7 with $k=4 \cdot|S|^{n} \cdot n$. Then, $R^{\prime} \subseteq H^{\prime} \times M$, and we may suppose that $\left|H^{\prime}\right|=4 \cdot|S|^{n} \cdot n \cdot|M|$.

Let $X \stackrel{\text { def }}{=} S^{n} \times M$, and $Y$ be a set with

(1) $|Y|=3 \cdot|X|$,

(2) $X \cap Y=\emptyset$,

and set $H \stackrel{\text { def }}{=} X \cup Y$. Thus, we can suppose that $H^{\prime}=n \times H$, and consequently $R^{\prime} \subseteq(n \times H) \times M$.

Choose two relations $Q \subseteq{ }^{2}(S \times Y), Q^{\prime} \subseteq{ }^{2} X$ such that

(1) $Q$ and $Q^{\prime}$ are symmetric, irreflexive, and rigid,

(2) The degree of every point in $Q$ or $Q^{\prime}$ is at most 5 .

Let $\sigma: S \times S \rightarrow S \times Y$ be a one-one function with the property that

$$
\sigma(s, z)_{0} \in C(s) \cap C(z)
$$

for all $s, z \in S$. Such a $\sigma$ exists, since $C(s)$ and $C(z)$ are big by Lemma 4.6.

Now, set $U \stackrel{\text { def }}{=} S \times H$, and define $R \subseteq U \times U$ as follows: Let $\langle s, u\rangle,\langle z, v\rangle \in U$. Then, we say that $\langle s, u\rangle R\langle z, v\rangle$ if and only if one of the following conditions holds:

(a) $s \prec z, v=\left\langle s_{0}, \ldots, s_{n-1}, m\right\rangle \in X, f\left(s_{0}, \ldots, s_{n-1}\right)=z$, and there is exactly one $i<n$ such that $s=s_{i}$ and $\langle i, u\rangle R^{\prime} m$,

(b) $z \prec s, u=\left\langle z_{0}, \ldots, z_{n-1}, m\right\rangle \in X, f\left(z_{0}, \ldots, z_{n-1}\right)=s$, and there is exactly one $i<n$ such that $z=z_{i}$ and $\langle i, v\rangle R^{\prime} m$,

(c) $s=z, u, v \in X, u Q^{\prime} v$,

(d) $s \in C(z), s \neq z$, and

(i) $u \in X, v \in Y,\langle z, v\rangle \notin \operatorname{ran}_{\sigma}(s)$, or

(ii) $u \in Y, v \in X,\langle s, u\rangle \notin \operatorname{ran}_{\sigma}(z)$.

(e) $v \in Y$ and $\langle s, u\rangle Q\langle z, v\rangle$.

From these conditions we immediately see that $R$ is symmetric and irreflexive. Also, 
Claim: For all $V \subseteq U$ with $|V|=n$, and for all $V^{\prime} \subseteq V$ there is some $u \in U$ such that for all $v \in V$,

$$
v R u \Longleftrightarrow v \in V^{\prime} .
$$

Proof of the claim: Enumerate $V$ by $\left\langle s_{0}, u_{0}\right\rangle, \ldots,\left\langle s_{n-1}, u_{n-1}\right\rangle$, and choose some $V^{\prime} \subseteq V$. Let $\left\{s_{0}, \ldots, s_{n-1}\right\} \subseteq\left\{z_{0}, \ldots, z_{n-1}\right\} \subseteq S$ with $\left|\left\{z_{0}, \ldots, z_{n-1}\right\}\right|=n$, and $f\left(s_{0}, \ldots, s_{n-1}\right)=z$. For each $j<n$ fix some $\nu(j) \in N$ with $s_{j}=z_{\nu(j)}$. Set $K \stackrel{\text { def }}{=}\left\{\left\langle\nu(j), u_{j}\right\rangle: j<n\right\}, K^{\prime} \stackrel{\text { def }}{=}\left\{\left\langle\nu(j), u_{j}\right\rangle: j<n,\left\langle s_{j}, u_{j}\right\rangle \in V^{\prime}\right\}$. Then, $|K|=n$ and $K^{\prime} \subseteq K \subseteq n \times H$.

Choose some $m \in M$ such that $x R^{\prime} m$ iff $x \in K^{\prime}$ for all $x \in K$, and let $q \stackrel{\text { def }}{=}$ $\left\langle z_{0}, \ldots, z_{n-1}, m\right\rangle$ and $p \stackrel{\text { def }}{=}\langle z, q\rangle$. Then, $q \in X$, and $s_{j} \prec z$ for each $j<n$.

By the conditions of Lemma 4.6 we have $z \nprec s_{j}$, if $s_{j} \neq z$. Thus, $\left\langle s_{j}, u_{j}\right\rangle R\langle z, q\rangle$ iff a. above holds.

Now, $\nu(j)$ is the unique index with $s_{j}=z_{\nu(j)}$, and therefore,

$$
\left\langle s_{j}, u_{j}\right\rangle R\langle z, q\rangle \Longleftrightarrow\left\langle\nu(j), u_{j}\right\rangle R^{\prime} m \Longleftrightarrow\left\langle s_{j}, u_{j}\right\rangle \in V^{\prime}
$$

by the properties of $m$. This proves the claim.

We next show that $R$ is rigid. Let $g$ be a base automorphism of $R$, and choose some $a \stackrel{\text { def }}{=}\langle s, u\rangle \in U$. Suppose that $u \in X$; then, by d. above,

$$
\operatorname{ran}_{R}(a) \supseteq(C(s) \times Y) \backslash\{\sigma(a, b): b \in S\},
$$

and therefore

$\left|\operatorname{ran}_{R}(a)\right| \geq|C(s)| \cdot|Y|-|S| \geq \frac{3}{4} \cdot|S| \cdot 3 \cdot|X|-|S|=\frac{9}{4} \cdot|S| \cdot|X|-|S|>2 \cdot|S| \cdot|X|$.

The last inequality follows from $|S|>5$ and $X=S^{n} \times M$, so that

$$
\frac{1}{4} \cdot|S| \cdot|X|>\frac{1}{4} \cdot|S| \cdot|S|^{n}>|S| .
$$

If $u \in Y$, then $\operatorname{ran}_{R}(a) \subseteq(S \times X) \cup F$, where $|F| \leq 5$, by a. - e. above. Hence,

$$
\left|\operatorname{ran}_{R}(a)\right| \leq|S| \cdot|X|+5<2 \cdot|S| \cdot|X| \text {. }
$$

This implies that $\operatorname{ran}(g\lceil(S \times X))=S \times X$, and $\operatorname{ran}(g\lceil(S \times Y))=S \times Y$. Since $Q$ is rigid, e. implies that $g\left\lceil(S \times Y) \subseteq i d_{U}\right.$.

Let $\langle s, u\rangle,\langle z, v\rangle \in U, u, v \in X, s \neq z$, and set $w \stackrel{\text { def }}{=} \sigma(s, z)$. Then, $w \notin \operatorname{ran}_{\sigma}(z)$, and thus $\langle\langle s, u\rangle, w\rangle \notin R$, but $\langle z, u\rangle R w$. Since $g(w)=w$ by $w \in S \times Y$, we have $\operatorname{ran}_{g}(\{s\} \times X)=\{s\} \times X$ for all $s \in S$.

Let $s \in S$ and $g^{\prime}: X \rightarrow X$ such that $g(s, u)=\left\langle s, g^{\prime}(u)\right\rangle$. Then, $g^{\prime}$ is a base automorphism of $Q^{\prime}$ by c., and therefore $g=i d_{X}$, since $Q^{\prime}$ is rigid. It follows that $g\left\lceil(S \times X) \subseteq i d_{U}\right.$ as well, and hence, $R$ is rigid.

Corollary 4.9. Suppose that $R$ is as in 4.8. The, the relations definable from $R$ with $n+1$ variables are exactly the unions of relations of the form

$$
\left\{s \in{ }^{n+1} U: \varphi_{i j}\left(s_{i}, s_{j}\right), i<j<n+1\right\},
$$

where $\varphi(x, y)$ is one of the formulas

$$
x=y, x \neq y, x R y, \neg x R y .
$$


Proof. Using the properties of $R$, this follows by an easy elimination of quantifiers argument.

By 4.8 and 4.9, $R$ is a rigid relation on a finite set $U$, with the property that not all subsets of $U$ are definable by $n+1$ variables.

For small sets, the answer to Jónsson's question is positive:

Proposition 4.10. If $|U| \leq 7$, then every rigid relation on $U$ generates $\mathfrak{R e l}(U)$. If $|U| \leq 9$, then every symmetric rigid relation on $U$ generates $\mathfrak{R e l}(U)$.

Proof. It was shown in [ADN95] that for $|U| \leq 6$, every subalgebra of $\mathfrak{R e r}(U)$ is Galois closed. Hence, if $R$ is rigid, then

$$
\langle R\rangle=\langle R\rangle^{\rho \sigma}=\mathfrak{R e l}(U)
$$

by 4.1. Now, let $|U|=7$, and $\mathfrak{A}$ be a proper subalgebra of $\mathfrak{R e l}(U)$; we shall show that $\mathfrak{A}$ is not rigid. First, consider the case that $\mathfrak{A}$ is not integral with intgral constituents $U_{i}, i<k$. By 2.3.3 (2) and the fact that no proper subalgebra of $\mathfrak{R e l}\left(U^{\prime}\right),\left|U^{\prime}\right| \leq 6$, is rigid, we can suppose that all constituent sets contain more than one element. In particular, since 7 is small, if $\left|U_{i}\right| \neq\left|U_{j}\right|$, then $\left|U_{i}\right|$ is not a multiple of $\left|U_{j}\right|$. We consider two cases:

1. $\left|U_{0}\right|=\left|U_{1}\right|=2,\left|U_{2}\right|=3$ : Clearly, for $i \in\{0,1\}$, any $P \in A t(\mathfrak{A}), P \subseteq U_{i} i$, and all $x, y \in U_{i}$ we have $\left|\operatorname{ran}_{P}(x)\right|=\left|\operatorname{ran}_{P}(y)\right|$. Thus, $U_{i} \times U_{2}$ and $U_{2} \times U_{i}$ are atoms of $\mathfrak{A}$. Let $M=U \backslash U_{2}$, and $\mathfrak{B}$ be the relative subalgebra of $\mathfrak{A}$ with respect to ${ }^{2} M$. Since the integral constituents of $\mathfrak{B}$ have more than element, and $|M|=4$, $\mathfrak{B}$ cannot be rigid. By 2.3.3, any base homomorphism of $\mathfrak{B}$ can be extended to $\mathfrak{A}$. It follows that $\mathfrak{A}$ is not rigid.

2. If $k=2$, then it is not hard to see that $U_{0} \times U_{1}$ is an atom of $\mathfrak{A}$. Since neither $\mathfrak{A}_{0}$ nor $\mathfrak{A}_{1}$ are rigid, $\mathfrak{A}$ is not rigid, again by 2.3.3.

Now, suppose that $\mathfrak{A}$ is integral. If $\mathfrak{A}$ contains a permutation $R$ which is not the identity, $R$ must move all points, and all cycles of $R$ must have the same cardinality. Since 7 is prime, $R$ generates the cyclic group of order 7 , thus $\mathfrak{A}=\langle R\rangle$ is not rigid.

Otherwise, $\mathfrak{A}$ has two, three or four atoms. If $\mathfrak{A}$ has two atoms, these are the identity and the diversity element, and $\mathfrak{A}$ is not rigid. If $\mathfrak{A}$ has three atoms, then there are two possible types, none of which is rigid on 7 elements, cf. [AM88]. Thus, suppose that $\mathfrak{A}$ has the four atoms $R, S, T$, and $1^{\prime}$. At least one of $R, S, T$ is symmetric, thus, suppose that $R=R^{-1}$. Since no $P \in\{R, S, T\}$ is a permutation, we have $\left|\operatorname{ran}_{P}(x)\right|=2$ for $P \in\{R, S, T\}$ and $x \in U$. If $\operatorname{ran}_{R}(a)=\operatorname{ran}_{R}(b)=$ $\{c, d\}$ for some $a, b \in U, a \neq b$, then it follows from $R=R^{-1}$ that $R$ has the incidence matrix

\begin{tabular}{|c||c|c|c|c|c|c|c|}
\hline$R$ & 0 & 1 & 2 & 3 & 4 & 5 & 6 \\
\hline \hline 0 & 0 & 0 & 1 & 1 & 0 & 0 & 0 \\
\hline 1 & 0 & 0 & 1 & 1 & 0 & 0 & 0 \\
\hline 2 & 1 & 1 & 0 & 0 & 0 & 0 & 0 \\
\hline 3 & 1 & 1 & 0 & 0 & 0 & 0 & 0 \\
\hline 4 & 0 & 0 & 0 & 0 & 0 & 1 & 1 \\
\hline 5 & 0 & 0 & 0 & 0 & 1 & 0 & 1 \\
\hline 6 & 0 & 0 & 0 & 0 & 1 & 1 & 0 \\
\hline
\end{tabular}


It is easy to check that $R$ generates a non integral algebra, so this case is not possible. Similarily, we see that $R$ cannot contain a $\mathrm{K}_{3}$, so that $(R \circ R) \cap R=\emptyset$, and that $\mathrm{R}$ is determined up to a permutation of $U$ in the following sense: If, in constructing the matrix of $R$, we always choose the smallest possible number $<7$, then we obtain $R$ as

\begin{tabular}{|c||c|c|c|c|c|c|c|}
\hline$R$ & 0 & 1 & 2 & 3 & 4 & 5 & 6 \\
\hline \hline 0 & 0 & 1 & 1 & 0 & 0 & 0 & 0 \\
\hline 1 & 1 & 0 & 0 & 1 & 0 & 0 & 0 \\
\hline 2 & 1 & 0 & 0 & 0 & 1 & 0 & 0 \\
\hline 3 & 0 & 1 & 0 & 0 & 0 & 1 & 0 \\
\hline 4 & 0 & 0 & 1 & 0 & 0 & 0 & 1 \\
\hline 5 & 0 & 0 & 0 & 1 & 0 & 0 & 1 \\
\hline 6 & 0 & 0 & 0 & 0 & 1 & 1 & 0 \\
\hline
\end{tabular}

We leave the straightforward details to the reader. $R$ is the union of the permutation $\varphi=(0135642)$ and its inverse, and it is easy to check that $\mathfrak{A}$ is not rigid.

A computer program kindly supplied to us by Brendan McKay (cf. [McK90]) found 3,696 non-isomorphic rigid graphs on eight elements, and 135,004 nonisomorphic rigid graphs on nine elements. RELALG ([Dün94]) then told us that each of these generates $\mathfrak{R e l}(U)$. Hence, our example of a rigid graph on 10 elements which does not generate all of $\mathfrak{R e l}(U)$ is minimal in the class of symmetric relations.

Problem 1. If $|U| \in\{8,9\}$, does every rigid relation on $U$ generate all of $\mathfrak{R e l}(U)$ ?

All the rigid relations we know on sets with at most 15 elements are 4-rigid. This gives rise to

Problem 2. Suppose that $|U|=n \geq 4$, and that $R \in \operatorname{Rel}(U)$ is rigid. Is every $P \in$ $\operatorname{Rel}(U)$ definable in $\langle U, R\rangle$ by a formula with $k$ variables, where $k=\left\lfloor\log _{2} n\right\rfloor+1$ ? In other words, is $R$ k-rigid?

Finally, let us turn to fragments of second order logic. $R$ is not rigid can be expressed by a $\Sigma_{1}^{1}$ sentence using only one extra binary predicate and three variables in the first order part:

$$
(\exists f)[f \text { is a non-identity permutation } \wedge f \circ R=R \circ f] .
$$

It is unknown whether rigidity is expressible in $\Sigma_{1}^{1}$. Monadic second order logic (MSO) seems a more hopeful candidate to express rigidity, but we shall show that this logic will not do, even with a built in linear order. We need one result due to N. Immerman, which appears here with his kind permission:

Proposition 4.11. Let $r, n<\omega$. Then, there are finite linearly ordered structures $\left\langle U_{0}, P_{0}\right\rangle,\left\langle V_{0}, Q_{0}\right\rangle$ which cannot be distinguished by sentences of MSO $+<$ having quantifier depth $r$ and using $n$ new unary predicates.

Proposition 4.12. Rigidity is not expressible in $M S O+<$.

Proof. Assume that rigidity can be expressed by an MSO $+<$ sentence having quantifier depth $r$ and using $n$ new unary predicates, and choose two linear orders $\left\langle U_{0}, P_{0}\right\rangle,\left\langle V_{0}, Q_{0}\right\rangle$ as in 4.11. Let $\left\langle U_{1}, P_{1}\right\rangle$ be isomorphic to $\left\langle U_{0}, P_{0}\right\rangle$, and let $\langle U, R\rangle$ be the disjoint union of these structures. Order $U$ by $x<_{U} y \stackrel{\text { def }}{\Longleftrightarrow}$ 
(1) $x, y \in U_{i}$ and $\langle x, y\rangle \in P_{i}$ for $i<2$, or

(2) $x \in U_{0}$ and $y \in U_{1}$.

Then, $R \subseteq<_{U}$, and $\mathrm{R}$ is not rigid on $U$, since the components of $R$ are linearly ordered by $R$ and have the same lengths. Similarly, let $\langle V, S\rangle$ be the disjoint union of $\left\langle U_{0}, P_{0}\right\rangle$ and $\left\langle V_{0}, Q_{0}\right\rangle$, and define the linear order $<_{V}$ on $V$ analogously to $<_{U}$. Then, $S$ is rigid on $V$, since the components have different lengths.

We now play the Ajtai-Fagin $(\mathrm{n}, \mathrm{r})$ - game of [FSV92]: The duplicator chooses $\langle U, R\rangle$ as the first structure and lets the spoiler colour it. Then, the duplicator chooses $\langle V, S\rangle$ as the second structure and colours it in such a way, that the components of $S$ match the components of $R$ in the way given by 4.11. In the subsequent Ehrenfeucht-Fraisse r-game, the duplicator always matches a pebble on one component of one structure with one from the corresponding component of the other structure. She wins the game restricted to the components by Immerman's result 4.11, and, since $U_{0}<_{U} U_{1}$ and $U_{0}<_{V} V_{0}$, and she always plays corresponding components, she can preserve the linear orders as well.

Finally, we shall see that non-rigidity is not expressible by a $\Sigma_{1}^{1}$ sentence where the first order part has s special form. The result has already been obtained via probabilistic arguments (cf. [Com88]); here we give a concrete construction:

Proposition 4.13. The property of being not rigid cannot be expressed by a $\Sigma_{1}^{1}$ sentence $\left(\exists P_{0}\right) \ldots\left(\exists P_{n-1}\right) \psi$, where $\psi$ is of the form $(\exists \bar{x})(\forall \bar{y}) \varphi$ and $\varphi$ is quantifier free.

Proof. Let $\chi$ be the sentence $(\exists R)(\exists x)(\exists y) \varphi$, where $R$ is a binary predicate symbol and $\varphi$ is the conjunction of the sentences 1.-6. below; we use the auxiliary unary predicates $U_{x}$ and $U_{y}$ with $U_{x}(z) \Leftrightarrow x=z \vee x R z$, and define $U_{y}$ analogously:

(1) $(\forall z) \neg z R z$

(2) $(\forall z)(\forall w)[z R w \rightarrow \neg w R z]$

(3) $(\forall u)(\forall v)(\forall w)[u R v \wedge v R w \rightarrow u R w]$

(4) $(\forall z)\left[\left(U_{x}(z) \vee U_{y}(z)\right) \wedge \neg\left(U_{x}(z) \wedge U_{y}(z)\right)\right]$

(5) $(\forall z)(\forall w)\left[U_{x}(z) \wedge U_{x}(w) \rightarrow z=w \vee z R w \vee w R z\right]$

(6) $(\forall z)(\forall w)\left[U_{y}(z) \wedge U_{y}(w) \rightarrow z=w \vee z R w \vee w R z\right]$

1.-3. say that $R$ is a strict partial order, 4. tells us that $R$ has exactly two connected components, and 5., 6. state that $R$ is a linear order on these components. $R$ is not rigid if and only if the components have the same cardinality.

Suppose that $\left(\exists P_{0}\right) \ldots\left(\exists P_{n-1}\right) \psi$, with $\psi$ having the prescribed form, says that $R$ is not rigid. Then, by possibly renaming variables, $\chi \wedge\left(\exists P_{0}\right) \ldots\left(\exists P_{n-1}\right) \psi$ is equivalent to a sentence of the desired form. Now,

$$
U \models \chi \wedge\left(\exists P_{0}\right) \ldots\left(\exists P_{n-1}\right) \psi \text { if and only if }|U| \text { is even. }
$$

It follows that the class of these formulas can express parity which is known not to be the case (cf. [Com88]).

It may be worthy of mention that, on the other hand, the property of being not rigid can be expressed by a $\Sigma_{1}^{1} \Pi_{2}^{0}$ sentence, since the property of a relation being a nonidentity base automorphism of $R$ is expressible by a prenex $(\forall \bar{x})(\exists \bar{y})$ sentence. 


\section{REFERENCES}

[ADN92] Hajnal Andréka, Ivo Düntsch, and István Németi, A non permutational integral relation algebra, Michigan Mathematical Journal 39 (1992), 371-384.

[ADN95] Hajnal Andréka, Ivo Düntsch, and István Németi, Binary relations and permutation groups, Mathematical Logic Quarterly, to appear (1995).

[AM88] Hajnal Andréka and Roger Maddux, Representations for small relation algebras, Preprint, Dept. of Mathematics, Iowa State University, 1988.

[BH79] A Blass and F Harary, Properties of almost all graphs and complexes, Journal of Graph Theory 3 (1979), 225-240.

[CK71] C. Chang and J. Keisler, Model theory, North Holland, Amsterdam, 1971.

[Com88] K J Compton, 0-1 laws in logic and combinatorics, Algorithms and order (Ivan Rival, ed.), Kluwer, 1988.

[Dün94] Ivo Düntsch, A microcomputer based system for small relation algebras, Journal of Symbolic Computation 18 (1994), 83-86.

[Fag87] Ron Fagin, Finite model theory - a personal perspective, Springer Lecture Notes in Computer Science 470 (1987), 3-24.

[FSV92] R Fagin, L Stockmeyer, and M Vardi, On monadic NP vs. monadic co-NP, Research report RJ 9225 (81789), IBM Almaden, 1992.

[HMT71] Leon Henkin, J. Donald Monk, and Alfred Tarski, Cylindric algebras, Part I, North Holland, Amsterdam, 1971.

[HMT85] Leon Henkin, J. Donald Monk, and Alfred Tarski, Cylindric algebras, Part II, North Holland, Amsterdam, 1985.

[IK89] Neil Immerman and Dexter Kozen, Definability with a bounded number of variables, Information and Computation 83 (1989), 121-139.

[Imm82] Neil Immerman, Upper and lower bounds for first order expressibility, Journal of Computer and System Science 25 (1982), 76-98.

[Jón84] Bjarni Jónsson, Maximal algebras of binary relations, Contemporary Mathematics 33 (1984), 299-307.

[JT52] Bjarni Jónsson and Alfred Tarski, Boolean algebras with operators II, American Journal of Mathematics 74 (1952), 127-162.

[KV92] P Kolaitis and M Vardi, Infinitary logic and 0-1 laws, Information and Computation 98 (1992), 258-294.

[McK66] Ralph McKenzie, Representations of integral relation algebras, Ph.D. thesis, University of Colorado, 1966.

[McK90] Brendan McKay, Nauty user guide, Tech. Report TR-CS-90-02, Australian National University, 1990.

[TG87] Alfred Tarski and Stephen Givant, A formalization of set theory without variables, American Math Society, New York, 1987.

(H. Andréka, I. Németi) Mathematical Institute, Academy of Sciences, Budapest 1363, HUNGARY 2

E-mail address: h2644ande @huella.bitnet

(I. Düntsch) School of INFORMATION AND SOFTWARE ENGINEERING, UNIVERSITY OF ULSTER at Jordanstown, NewtownabBey, BT 37 0QB, N. Ireland ${ }^{3}$

E-mail address: I.Duentsch@ulst.ac.uk

\footnotetext{
${ }^{2}$ Andréka's and Németi's research sponsored by Hungarian National Foundation for Scientific Research Grants 1911 and T7255

${ }^{3}$ Also Institut für semantische Informationsverarbeitung, Universität Osnabrück
} 